\title{
The effect of vitality and dentin depth on resin tags length of resin-dentin interface in dogs' teeth
}

\author{
Mohamed Sanad ${ }^{1}$ and Mohammed H. Shekidef2,* \\ ${ }^{1}$ Department of Conservative Dentistry, Faculty of Dentistry, Suez Canal University, Ismailia, Egypt \\ ${ }^{2}$ Department of Surgery, Anesthesiology and Radiology, Faculty of Veterinary Medicine, Suez Canal University, \\ Ismailia, Egypt
}

\begin{abstract}
The aim of this study was to evaluate the effect of pulpal pressure and dentin depth on the quality of hybrid layer of bonded composite. Four healthy dogs, aged between 12 and 18 months, with intact dentitions were used for the experimental work. A total of 24 teeth were available for the study by using the six upper anterior teeth of each of the four dogs used in the study. The 24 teeth included in the study were divided according to presence or absence of pulpal pressure $(P)$ into two groups with 12 teeth in each group. $P_{1}$ teeth with positive pulpal pressure and $P_{2}$ teeth with no pulpal pressure. Each of these two groups was further divided into two subgroups, according to the cavity depth $(D)$ they received, where in $D_{1}$ a class $\mathrm{V}$ cavity with depth of $1 \mathrm{~mm}$ was performed, while in $D_{2}$ a class $\mathrm{V}$ cavity with depth of $2 \mathrm{~mm}$ was performed. The cavities were restored with micro hybrid composite restorative material and the samples were then left in the dogs' mouth for 30 days. At the end of the experimental period, the dogs were euthanized by injecting an overdose of thiopental sodium. The anterior part of the upper jaw was sawed out of each dog's mouth and the teeth were sectioned to evaluate the resin-dentin interface using Environmental Scanning Electronic Microscope. Results of the study showed that the mean resin tag length value was significantly affected by vitality and dentin depth. The presence of pulpal pressure together with depth of dentin could adversely affect the quality of hybridization.
\end{abstract}

Keywords: Dentin, Dogs, Resin tags, Teeth.

\section{Introduction}

The successful bonding to tooth structures is imperative for retention, marginal adaptation, and durability of tooth colored restorations. Although bonding to enamel is a relatively simple procedure, without major technical requirements or difficulties, bonding to dentin represents a much greater challenge, which may lead to clinical failure of restorations (Jacques and Hebling, 2005).

Dentin is a dynamic tissue with a complex structure. A serum like fluid fills the dentinal tubules, which flows from the pulp chamber under a hydrodynamic pressure of approximately $24 \mathrm{~cm} \mathrm{Hg}$ or $32.5 \mathrm{~cm} \mathrm{H}_{2} \mathrm{O}$ in vital teeth (Oskoee et al., 2009). The water content of the tubular fluid interferes with polymerization reaction of resinous adhesives resulting in suboptimal conversion rates (Moll et al., 2005). For optimal bonding to dentin, the consequence of outward flow through the dentinal tubules due to the positive pulpal pressure should be taken into consideration (Montanari et al., 2007).

In addition to the effect of pulpal pressure on resin bonding, regional variations in dentin morphology at different dentin depths may affect the quality of bonding (Hebling et al., 2007). Dentin is a complex substrate composed of dentinal tubules, intertubular dentin, and peritubular dentin. The dentinal tubule density in a 2-mm thick dentin bridge is typically 30,000 tubules per square millimeter. The number of tubules per square millimeter is increased more than three times from the dentino-enamel junction to the pulp in the coronal dentin (Yoshikawa et al., 2012). Bonding to deep dentin has been more challenging than bonding to superficial dentin mainly due to the reduced area of solid intertubular dentin associated with the increased water content.

It was noted that most of the studies investigating the effect of pulpal pressure were conducted in vitro by pulpal pressure simulation devices, thus it was found interesting to study the effect of pulpal pressure and dentin depth on the quality of hybrid layer in vivo on dogs' teeth to simulate the clinical situation where both factors apply together in the oral environment.

\section{Materials and Methods}

A total of 24 upper anterior teeth of four healthy dogs, aged between 12 and 18 months, with intact dentitions were used for the experimental work. The experimental protocol on dogs was conducted according to the ethical guidelines for animal care in the Faculty of Veterinary Medicine, Suez Canal University.

The 24 teeth included in the study were divided according to presence or absence of pulpal pressure $(P)$ into two groups with 12 teeth in each group, where in $P_{1}$ teeth had positive pulpal pressure, while in $P_{2}$ 
teeth had no pulpal pressure. Each of these two groups was further divided into two subgroups, six teeth each, according to the cavity depth $(D)$ they received, where in $D_{1}$ a class $V$ cavity with depth of $1 \mathrm{~mm}$ was performed, while in $D_{2}$ a class $\mathrm{V}$ cavity with depth of 2 mm was performed. The samples were left in the dogs' mouths for 30 days.

Before preparation, each animal was premedicated with an intramuscular injection of $1 \mathrm{mg} / \mathrm{kg}$ chloropromazine hydrochloride (Misr Co. Pharm. Industries, S.A.A.). General anesthesia was then induced with an intravenous injection of thiopental sodium solution 2.5\% (Sandoz GmbH, Kundl-Austria) until the main reflexes were abolished (Hall et al., 2001). All teeth were scaled and polished with a rubber cup, on the day of operative procedure. Teeth were isolated using sterile cotton rolls and saliva was controlled through high-volume evacuation.

In each dog, the right upper anterior teeth were devitalized at first to exclude pulpal pressure, while the left side had its vitality preserved. After isolation of the teeth and disinfection of the operative field with $2 \%$ chlorhexidine gluconate, palatal access to the pulp chamber was made using a round carbide bur, the coronal pulp tissue was then extirpated using a spoon excavator and the working length was determined by an apex locator (DentaPort Root ZX, Morita, Tokyo, Japan). The root canals were instrumented using K-files (Mani, Inc., Tochigi, Japan) up to size \#40 (Fig. 1) under irrigation with normal saline solution at each instrument change (Leonardo et al., 2007).

After final irrigation, the root canals were dried with sterile paper points and then the access cavities were filled with a glass ionomer cement (Vitremer; 3M ESPE, St Paul, MN) base to avoid entry of any food debris into the cleaned root canals.

Standardized Class V cavity preparations (cylindrical cavities with 2-mm diameter and either 1.0 or $2.0 \mathrm{~mm}$ deep) were prepared on the buccal surface of all teeth in each assigned group using round carbide burs (FG-2, Kerr; Karlsruhe, Germany) in a high-speed handpiece with a copious water spray (Taha et al., 2012) (Fig.

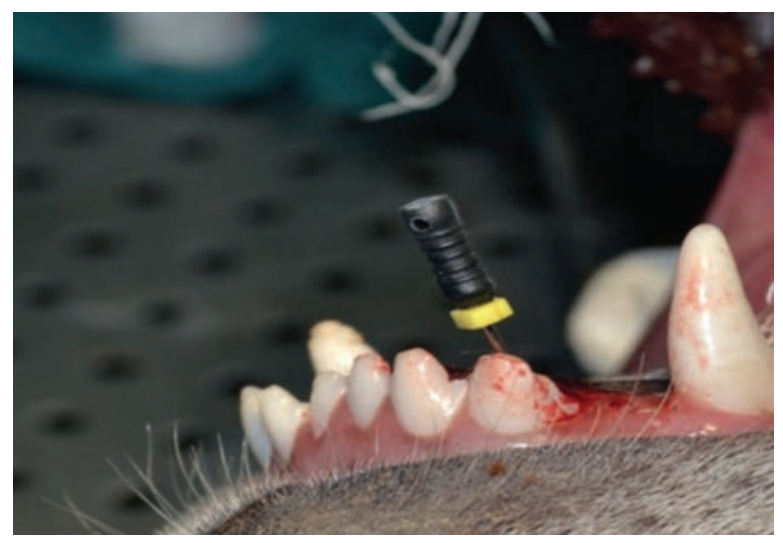

Fig. 1. Cleaning and shaping up to file $\# 40$.
2), the pulpal floor was flattened by an inverted cone carbide bur (FG-34, Kerr; Karlsruhe, Germany) and the side walls were finally finished by a fissure carbide bur (FG-56, Kerr; Karlsruhe, Germany). A digital caliper was used for standardizing the cavity diameter (Mondelli et al., 2009), while the cavity depth was checked using periodontal probe (Fig. 3). New burs were used after every five cavities in order to insure high cutting efficiency (Plotino et al., 2008). The retention relied only on the adhesion of resin composite to tooth structure where all the walls were prepared perpendicular to the axial wall (no undercuts) (Deliperi and Bardwell, 2009; Monga et al., 2009). All the internal line angles were rounded (Plotino et al., 2008). For restoration of the prepared cavities, each cavity wall was etched with $37 \%$ phosphoric acid etching gel (Scotchbond ${ }^{\mathrm{TM}}$ Etchant, 3M ESPE, St Paul, MN) (Fig. 4) for 20 seconds and then rinsed with water spray for 30 seconds to ensure complete removal of the etching gel byproducts (Deliperi and Bardwell, 2009). After rinsing and blotting excess moisture from the preparation using a cotton pellet, complete isolation for the teeth was performed using cotton rolls and then dentin bonding agent (Adper TM Single Bond 2, 3M ESPE, St Paul, MN) was applied in two layers to the cavities with a micro brush according to the manufacturer's instructions (Fig. 5). An air flow for 2-5 seconds was applied and the bonding agent was light cured for 20 seconds using blue-phase light curing unit with an output power of $800 \mathrm{~mW} / \mathrm{cm}^{2}$ power density (Bluephase C8, Ivoclar Vivadent). All light curing procedures were performed from a labial direction.

The class $\mathrm{V}$ preparations were then restored with micro hybrid composite restorative material (Z250 Filtek TM, 3M ESPE, St Paul, MN) (Fig. 6). Each layer was light cured for 20 seconds (according to the manufacturer's instructions) with the same light curing unit and the same power density. The curing distance was standardized through applying the tip of the curing unit on the labial surface of the teeth. The resin composite restorations were finally finished using a fine tapered finishing stone (TR-11 EF, Mani, Inc., Tochigi, Japan)

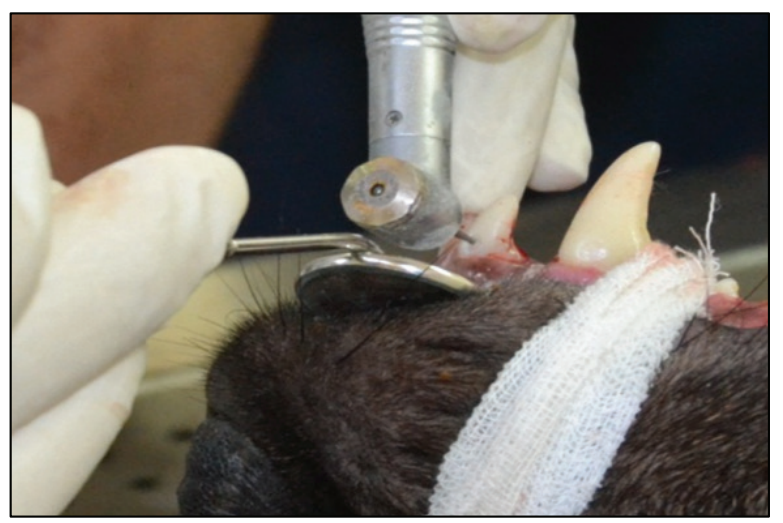

Fig. 2. Class V cavity preparation in upper anterior tooth. 


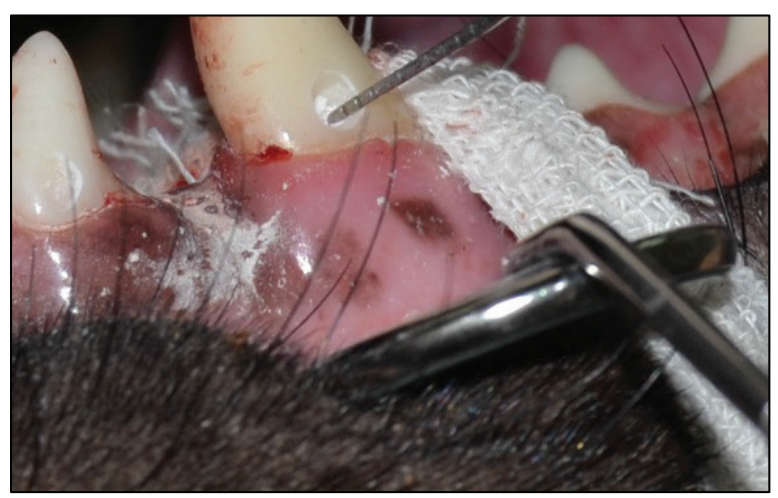

Fig. 3. Verification of the cavity depth using a periodontal probe (2 mm).

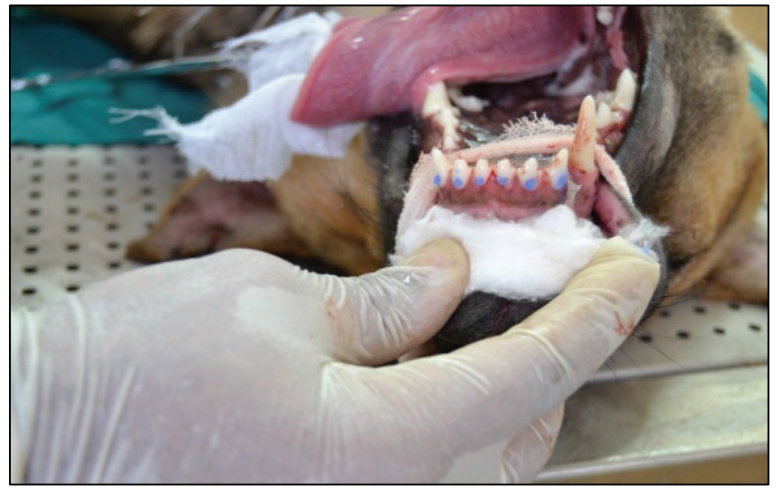

Fig. 4. Etching of cavity walls with $37 \%$ phosphoric acid.

to obtain a smooth surface of the restoration flushed with the tooth surface.

The four dogs were left for 30 days. During this period, all dogs were fed a soft diet to avoid fracture of the weakened devitalized teeth (Shipper et al., 2005).

At the end of the experimental period, the dogs were euthanized by injecting an overdose of thiopental sodium (Abd El Halim, 2011). The anterior part of the upper jaw was sawed out of each dog's mouth and the teeth were sectioned below the level of the cementoenamel junction using a diamond disc under constant irrigation, taking the necessary care not to traumatize the restored area (Fig. 7). The coronal portion of each tooth was then sectioned longitudinally through the restoration in a labiolingual plane to evaluate the resin-dentin interface. One section was made per tooth, producing two specimens.

Prior to teeth examination, teeth were placed in hydrochloric acid ( $\mathrm{HCl}$ ) for $1 \mathrm{~min}$ (Gordan et al., 1998) and then in sodium hypochlorite $(\mathrm{NaOCl})$ for $10 \mathrm{~min}$ (Lotfi et al., 2012) in order to remove the smear layer and the organic component of dentin, respectively.

The hybrid layer along the interface between the restoration and dentin was scanned using Environmental Scanning Electronic Microscope (Quanta200-FEIcollected at Netherland) and the obtained images were

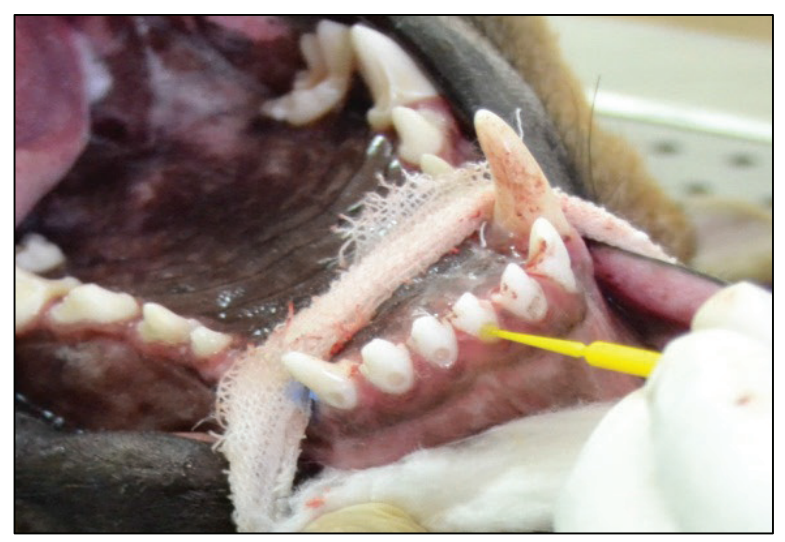

Fig. 5. Application of bonding agent after etching and rinsing.

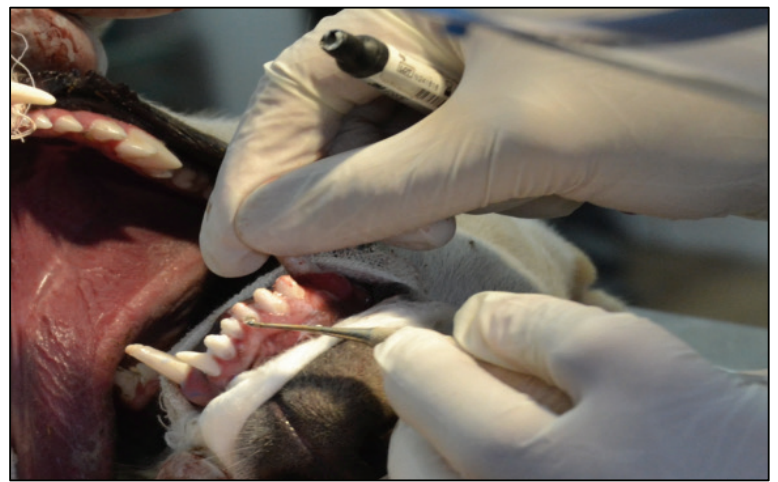

Fig. 6. Restoration of the cavities with resin composite.

then analyzed using specialized software (Semafore 5.21, Insinooritoimisto, Rimppi Oy, Finland) (Figs. 8-11).

Results were recorded, tabulated, and statistically analyzed using SPSS software program version 14 . Data were presented as mean and standard deviation. The significance level was set at $p \leq 0.05$.

\section{Results}

\section{Effect of vitality on resin tag length}

Data in Table 1 represent descriptive statistics and test of significance using T-independent test showing the effect of vitality on resin tag length of bonded resin composite to dentin using an etch and rinse bonding system (Single bond 2). The data revealed that the mean resin tag length value was higher in non-vital groups $(30.04 \mu \mathrm{m})$ than that of vital groups $(22.78 \mu \mathrm{m})$. Statistical analysis using T-independent test revealed that the difference was statistically significant $(p<$ $0.05)$.

\section{Effect of dentin depth on resin tag length}

Data in Table 2 represent descriptive statistics and test of significance using T-independent test showing the effect of dentin depth on resin tag length of bonded resin composite to dentin using an etch and rinse bonding system (Single bond 2). The data revealed that the mean resin tag length value was lower at $2 \mathrm{~mm}$ dentin 


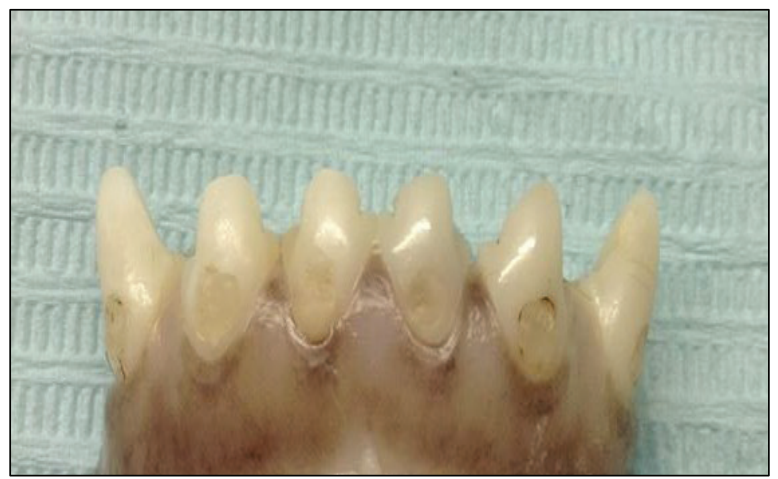

Fig. 7. Upper jaw after being sectioned from the dog's mouth.

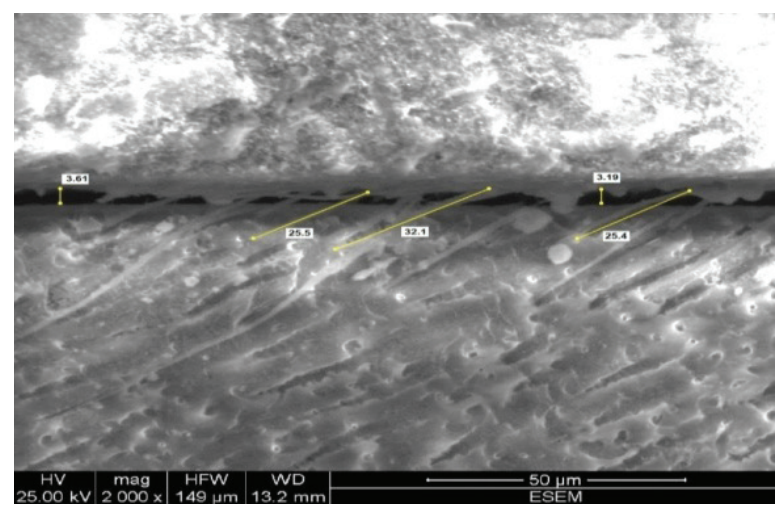

Fig. 8. An electron microscopic evaluation of a specimen from group $P_{1} D_{1}$ showed a clearly recognizable thin hybrid layer, which was, defined and continuous. However, adhesive failure was seen at the resin-dentin interface creating a number of gaps. Long resin tags were seen with the average length of $27.6 \mu \mathrm{m}$.

depth $(23.50 \mu \mathrm{m})$ than that at $1 \mathrm{~mm}$ dentin depth $(29.33$ $\mu \mathrm{m})$. Statistical analysis using T-independent test revealed that the difference was statistically significant $(p<0.05)$.

\section{Interactive effect of vitality and dentin depth on resin} tag length

Data in Table 3 represent descriptive statistics and test of significance using one-way-analysis of variance (ANOVA) showing the effect of interaction of vitality and dentin depth on resin tag length of bonded resin composite to dentin using an etch and rinse bonding system to dentin.

Statistical analysis using one-way-ANOVA revealed that at each dentin depth, the resin tag length value was significantly lower in vital groups than non-vital groups $P_{1} D_{1}(27.8 \mu \mathrm{m})$ versus $P_{2} D_{1}(30.86 \mu \mathrm{m})$ and $P_{1} D_{2}$ $(17.77 \mu \mathrm{m})$ versus $P_{2} D_{2}(29.23 \mu \mathrm{m})$. On the other hand, statistical analysis revealed that resin tag length value was significantly affected by dentin depth only in vital groups $P_{1} D_{1}(27.8 \mu \mathrm{m})$ versus $P_{1} D_{2}(17.77 \mu \mathrm{m})$, while in non-vital groups the depth was statistically nonsignificant $P_{2} D_{2}(29.23 \mu \mathrm{m})$ versus $P_{2} D_{1}(30.86 \mu \mathrm{m})$.

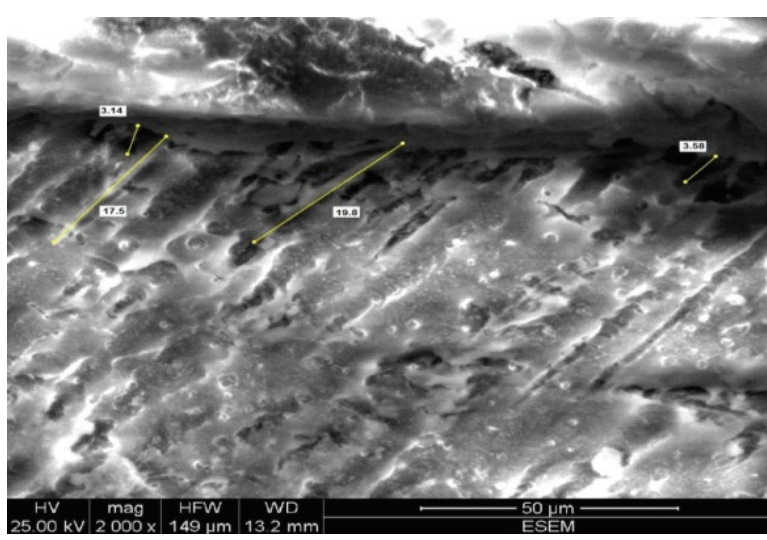

Fig. 9. An electron microscopic evaluation of a specimen from group $P_{1} D_{2}$ showed an interrupted hybrid layer with multiple voids. Thin and conical resin tags were observed with no evidence of lateral branching. The mean length of the resin tags was $18.63 \mu \mathrm{m}$. Areas of adhesive failure can be seen at the resin-dentin interface creating a number of gaps. Areas of cohesive failure were also seen within the resin and the resin-composite interface.

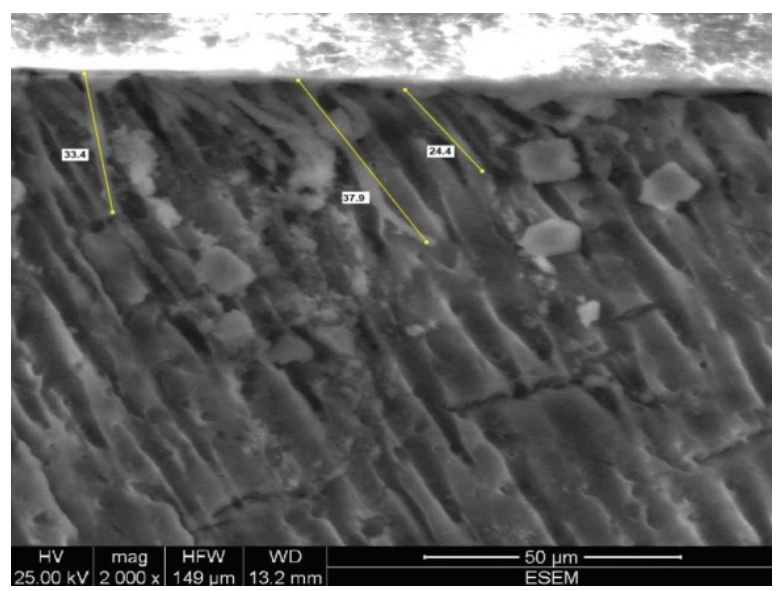

Fig. 10. An electron microscopic evaluation of a specimen from group $\mathrm{P}_{2} \mathrm{D}_{1}$ showed a clearly recognizable thin hybrid layer. There were few areas where there was a visible region of separated layers showing adhesive failure between the dentin and resin interface. The mean length of the resin tags was $31.18 \mu \mathrm{m}$. The resin tags were thick and bulbous, showing evidence of lateral tags.

\section{Discussion}

In this study, the effect of pulpal pressure and dentin depth on the quality of hybridization of composite resin were investigated in vivo, on dogs' teeth to simulate the effect of these factors in the oral environment. The dog model was chosen due to the close similarity to humans in the pulpal tissues and apical and periapical healing processes (Weinberg and Bral, 1999).

The results of the current study revealed that the quality of bond in terms of resin tag length within the hybrid layer of an etch and rinse bonding system to dentin was 


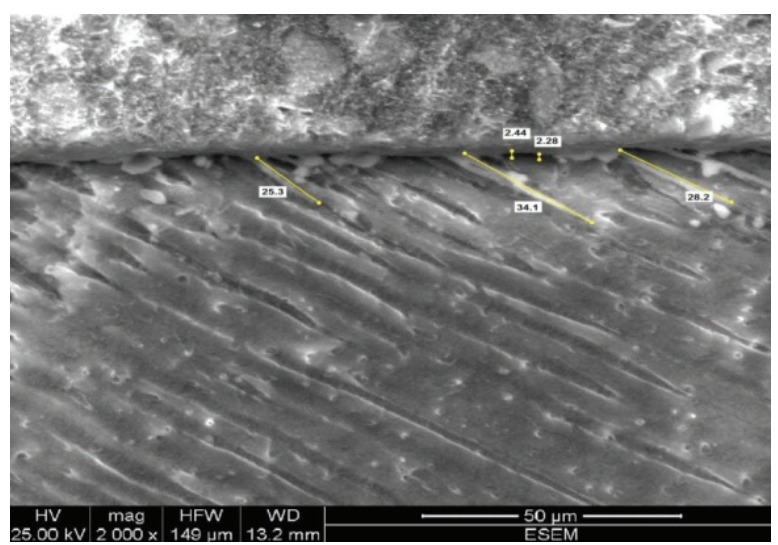

Fig. 11. An electron Microscopic evaluation of a specimen from group $P_{2} D_{2}$ showed a clearly recognizable hybrid layer which was thin and continuous. Areas of adhesive failure can be seen at the resin-dentin interface. The resin tags were thin and pointed. The mean length of the resin tags was $29.18 \mu \mathrm{m}$.

Table 1. Mean and standard deviation of resin tag length values representing the effect of vitality.

\begin{tabular}{lccc}
\hline Vitality & $\begin{array}{c}\text { Mean in } \\
\text { microns }(\boldsymbol{\mu m})\end{array}$ & $\begin{array}{c}\text { Standard } \\
\text { deviation }\end{array}$ & Significance \\
\hline Vital $\left(P_{1}\right)$ & 22.78 & 5.31 & 0.0001 \\
\hline Non vital $\left(P_{2}\right)$ & 30.04 & 2.46 & \\
\hline
\end{tabular}

significantly affected by the presence or loss of vitality with subsequent alteration of intrapulpal pressure at the two different dentin depths. In general, longer resin tags bunched together with lateral branching were found only in the case of dropped intrapulpal pressure due to loss of vitality.

Intrapulpal pressure has been described as one of the factors able to negatively interfere with dentin adhesion (Leloup et al., 2001). The pulpal pressure in vital teeth is not the same in different clinical and physiological situations. The average value of this pressure has been reported to be about $30-40 \mathrm{~cm} \mathrm{H}_{2} \mathrm{O}$ in normal physiologic conditions (Gupta and Tewari, 2006), as a result of this pressure the increased outward flow of tubular fluid after smear layer removal has been described to variably counteract the penetration of resin monomers to dentin surfaces dependent on the type of monomers used (Hebling et al., 2007).

On the other hand, in devitalized teeth samples, the absence of intrapulpal pressure led to decreased tubular fluid outflow, more desirable water condition necessary for adequate bonding and more efficient resin infiltration for the etched dentinal tubules.

The results of this part of the study were in agreement with the results of Cardoso et al. (2008) who reported less evident resin tag formation in the presence of intrapulpal pressure simulation. In addition, the results were parallel to the results of previous studies in which bond strength to bur cut dentin was decreased via pulpal
Table 2. Mean and standard deviation of resin tag length values representing the effect of dentin depth.

\begin{tabular}{cccc}
\hline Dentin depth & $\begin{array}{c}\text { Mean in } \\
\text { microns }(\boldsymbol{\mu m})\end{array}$ & $\begin{array}{c}\text { Standard } \\
\text { deviation }\end{array}$ & Significance \\
\hline $1 \mathrm{~mm}\left(D_{1}\right)$ & 29.33 & 2.58 & 0.0000 \\
\hline $2 \mathrm{~mm}\left(D_{2}\right)$ & 23.50 & 6.08 & \\
\hline
\end{tabular}

Table 3. Mean of resin tag length values representing the effect of depth and vitality.

\begin{tabular}{ccc}
\hline \multirow{2}{*}{ Dentin depth } & \multicolumn{2}{c}{ Vitality } \\
\cline { 2 - 3 } & Vital $\left.\mathbf{( P}_{\mathbf{1}}\right)$ & Non vital $\mathbf{( P}_{\mathbf{2}} \mathbf{)}$ \\
\hline $1 \mathrm{~mm}\left(D_{1}\right)$ & $27.8 \mu \mathrm{m}^{(\mathrm{b})}$ & $30.86 \mu \mathrm{m}^{(\mathrm{a})}$ \\
$2 \mathrm{~mm}\left(D_{2}\right)$ & $17.77 \mu \mathrm{m}^{(\mathrm{c})}$ & $29.23 \mu \mathrm{m}^{(\mathrm{a})}$ \\
\hline \multicolumn{2}{c}{1.71} \\
\hline
\end{tabular}

Means with the same alphabetical letters are not statistically significant while different letters indicate statistically significant difference.

pressure simulation (Tay et al., 2000; Moll et al., 2005; Gupta and Tewari, 2006; Sauro et al., 2007).

On the other hand, the results of this study do not agree with Brulat et al. (2008) who did not find any difference of bond strength of different adhesive systems to laser prepared dentin in absence or presence of pulpal pressure. The differences between their results and this study may be related to the difference in technique of cavity preparation and adhesives used. All-in-one and self-etch adhesive systems used in their study may have not been able to remove the damaged superficial layer of dentin and attached melted collagen fibers produced by laser ablation, with resultant reduction in tubular fluid even in presence of pulpal pressure.

The results of the current study showed the lower qualities of hybrid layer in terms of resin tag length in deep dentin when compared with those produced in superficial dentin; however, the differences in mean resin tag length reached statistical significance only in the presence of intrapulpal pressure, demonstrating the synergistic effect of both parameters (Table 3).

Regional variations in dentin morphology also play an important role in determining the quality of hybrid layer. Variations in tubule density and areas of intertubular dentin may modify the bond of both total-etch and self-etching systems (Hebling et al., 2007). Even in the absence of intrapulpal pressure, bonding to deep dentin has been more difficult to accomplish, where the increase in dentinal tubule diameter and density per millimeter together with the reduction in solid intertubular dentin compromise the bond (Giannini et al., 2001).

Although it has been indicated that moisture on the dentin surface is essential for a successful bonding protocol, an over-wet condition may contribute negatively to the adhesive procedure (Kanca, 1992). The advantages of a wet bonding technique are related 
to the ability of water to keep the demineralized collagen network open during primer infiltration (Tay et al., 2000). However, with increased dentin depth, excessive amounts of water related to the presence of intrapulpal pressure and wider dentinal tubules may be generated. The establishment of resin-dentin bonds in deep dentin under positive intrapulpal pressure challenges the balance between the appropriate amounts of water to warrant the creation of a well infiltrated hybrid layer and the negative effects of excessive wetness (Cardoso et al., 2008). This is particularly true in total etch systems, which beside completely removing the smear layer and smear plug also enlarge the entrance of dentinal tubules by removing peritubular dentin, increasing both dentin wetness and hydraulic conductance.

However, in case of absence of pulpal pressure, the volume of dentinal fluid permeated through dentin to the exposed surface was apparently not profuse enough to cause detrimental effects on resin infiltration, even in deep dentin what is in line with the studies performed by Yoshiyama et al. (1995) and Toledano et al. (2003). In the current experiment, the used etch and rinse bonding agent is an ethanol/water-based hydrophilic bonding agent; consequently, it is able to efficiently infiltrate the demineralized dentin in the presence of water provided that intrapulpal pressure is absent or reduced.

The results of this part of the study came in agreement with Hebling et al. (2007) who reported lower bond strength in deep dentin when applied in positive pulpal pressure. The results also coincided with Anido-Anido et al. (2012) who reported lower bond strengths at increased dentin depths.

On the contrary, the results of this study oppose to Toledano et al. (2003) who reported higher bond strengths for ethanol and acetone-based adhesive systems when applied in deep dentin, concluding that bond strength to dentin is both adhesive and substrate dependent. The difference may be due to the fact that the current study was conducted in vivo, so bonding was affected by presence of intrapulpal pressure in addition to different depths.

In conclusion, the presence of pulpal pressure together with depth of dentin could adversely affect the quality of hybridization.

\section{References}

Abd El Halim, S.A. 2011. Environmental scanning electron microscope study of enamel surface after using three different bleaching agents. J. Am. Sci. $7,11$.

Anido-Anido, A., Amore, R., Lewogy, H.R. and Anaute-Netto, C. 2012. Comparative study of bond strength to human and bovine dentin at three different depths. Braz. Dent. Sci. 15, 56-62.

Brulat, N., Leforestier, E., Rocca, J.P., Darquet-Cerretti, E. and Bertrand, M.F. 2008. Shear bond strength of self-etching adhesive systems to Er:YAG laserprepared dentine with and without pulpal pressure simulation. Photomed. Laser Surg. Dec. 26, 579-583.

Cardoso, M.V., Moretto, S.G., Carvalho, R.C. and Russo, E.M. 2008. Influence of intrapulpal pressure simulation on the bond strength of adhesive systems to dentin. Braz. Oral Res. 22, 170-175.

Deliperi, S. and Bardwell, D.N. 2009. Reconstruction of nonvital teeth using direct fiber-reinforced composite resin: a pilot clinical study. J. Adhes. Dent. 11, 71-78.

Giannini, M., Carvalho, R.M., Martins, L.R.M., Dias, C.T.S. and Pashley, D.H. 2001. The influence of tubule density and area of solid dentin on bond strength of two adhesive systems to dentin. J. Adhes. Dent. 3, 315-324.

Gordan, V.V., Vargas, M.A. and Denehy, G.E. 1998. Interfacial ultrastructure of the resin-enamel region of three adhesive systems. Am. J. Dent. 11, 13-16.

Gupta, R. and Tewari, S. 2006. Effect of rotary instrumentation on composite bond strength with simulated pulpal pressure. Oper. Dent. 31, 188-196.

Hall, L.W., Clarke, K.W. and Trim, C.M. 2001. Principles of sedation, analgesia and premedication in veterinary anesthesia $10^{\text {th }}$ edition. London, UK: W.B. Saunders.

Hebling, J., Castro, F.L. and Costa, C.A. 2007. Adhesive performance of dentin bonding agents applied in vivo and in vitro. Effect of intrapulpal pressure and dentin depth. J. Biomed. Mater. Res. B. Appl. Biomater. 83, 295-303.

Jacques, P. and Hebling, J. 2005. Effect of dentin conditioners on the microtensile bond strength of a conventional and a self-etching primer adhesive system. Dent. Mater. 21, 103-109.

Kanca, J. 3rd. 1992. Effect of resin primer solvents and surface wetness on resin composite bond strength to dentin. Am. J. Dent. 5, 213-215.

Leloup, G., D’Hoore, W., Bouter, D., Degrange, M. and Vreven, J. 2001. Meta-analytical review of factors involved in dentin adherence. J. Dent. Res. 80, 1605-1614.

Leonardo, M.R., Barnett, F., Debelian, G.J., de Pontes Lima, R.K. and Bezerra da Silva, L.A. 2007. Root canal adhesive filling in dogs' teeth with or without coronal restoration: a histopathological evaluation. J. Endod. 33, 1299-1303.

Lotfi, M., Moghaddam, N., Vosoughhosseini, S., Zand, V. and Saghiri, M.A. 2012. Effect of duration of irrigation with sodium hypochlorite in clinical protocol of MTAD on removal of smear layer and creating dentinal erosion. J. Dent. Res. Dent. Clin. Dent. Prospects. 6, 79-84.

Moll, K., Park, H.J. and Haller, B. 2005. Effect of simulated pulpal pressure on dentin bond strength of self-etching bonding systems. Am. J. Dent. 18, 335-339.

Mondelli, R.F., Ishikiriama, S.K., de Oliveira Filho, O. and Mondelli, J. 2009. Fracture resistance of weakened teeth restored with condensable resin 
with and without cusp coverage. J. Appl. Oral. Sci. $17,161-165$.

Monga, P., Sharma, V. and Kumar, S. 2009. Comparison of fracture resistance of endodontically treated teeth using different coronal restorative materials: an in vitro study. J. Conserv. Dent. 12, 154-159.

Montanari, M., Sauro, S., Pashley, D.H., Chersoni, S., Carvalho, R.M., Toledano, M., Osorio, R., Tay, F.R. and Prati, C. 2007. Effect of simulated pulpal pressure on dentin permeability and adhesion of self-etch adhesives. Dent. Mater. 23, 705-713.

Oskoee, S.S., Oskoee, P.A., Kimyai, S., Mohammadi, N. and Rikhtegaran, S. 2009. Effect of simulated pulpal pressure on composite bond strength to dentin prepared using Er, Cr: YSGG laser. Afr. J. Biotech. 8, 5547-5551.

Plotino, G., Buono, L., Grande, N.M., Lamorgese, V. and Somma, F. 2008. Fracture resistance of endodontically treated molars restored with extensive composite resin restorations. J. Prosthet. Dent. 99, 225-232.

Sauro, S., Pashley, D.H., Montanari, M., Chersoni, S., Carvalho, R.M., Toledano, M., Osorio, R., Tay, F.R. and Prati, C. 2007. Effect of simulated pulpal pressure on dentin permeability and adhesion of self-etch adhesives. Dent. Mater. 23, 705-713.

Shipper, G., Teixeira, F.B., Arnold, R.R. and Trope, M. 2005. Periapical inflammation after coronal microbial inoculation of dog roots filled with guttapercha or resilon. J. Endod. 31, 91-96.

Taha, N.A., Palamara, J.E and Messer, H.H. 2012. Assessment of laminate technique using glass ionomer and resin composite for restoration of root filled teeth. J. Dent. 40, 617-623.

Tay, F.R., Sano, H., Carvalho, R., Pashley, E.L. and Pashley, D.H. 2000. An ultrastructural study of the influence of acidity of self-etching primers and smear layer thickness on bonding to intact dentin. J. Adhes. Dent. 2, 83-98.

Toledano, M., Osorio, R., Ceballos, L., Fuentes, M.V., Fernandes, C.A., Tay, F.R. and Carvalho, R.M. 2003. Microtensile bond strength of several adhesive systems to different dentin depths. Am. J. Dent. 16, 292-298.

Weinberg, M.A. and Bral, M. 1999. Laboratory animal models in periodontology. J. Clin. Periodontol. 26, 335-340.

Yoshikawa, T., Wattanawongpitak, N., Cho, E. and Tagami, J. 2012. Effect of remaining dentin thickness on bond strength of various adhesive systems to dentin. Dent. Mater. J. 31, 1033-1038.

Yoshiyama, M., Carvalho, R.M., Sano, H., Horner, J.A., Brewer, P.D. and Pashley, D.H. 1995. Interfacial morphology and strength of bonds made to superficial versus deep dentin. Am. J. Dent. 8, 297-302. 